\title{
Evaluación de los significados personales en la construcción de la función lineal'
}

\author{
Assessing personal meanings in the construction of the linear function \\ Avaliando os significados pessoal na construção da função linear
}

Recibido: mayo de 2013

Aprobado: agosto de 2013
Fabio Nelson Torres Zuñeda ${ }^{2}$

Orlando Lurduy Ortegón ${ }^{3}$

\section{Resumen}

En este documento se tratan los principales aspectos para el diseño de una investigación que evalúa los significados personales de los estudiantes de grado noveno en la construcción noción de función lineal. Inicia con los ejes estructurales del trabajo de grado, la revisión teórica y se expone la teoría base del trabajo Enfoque Ontosemiótico de la Instrucción y la Cognición Matemática.

En el último apartado, se ubica la propuesta de investigación bajo el método de investigación Cualitativo y se exponen las fases que se tendrán en cuenta: partiendo de la delimitación del enfoque teórico, implementación de una secuencia de actividades, construcción de instrumentos de caracterización y análisis, triangulación de los análisis obtenidos, hasta llegar al informe final.

Palabras clave: Enfoque Ontosemiótico; Objeto matemático; Facetas institucional y personal; Elementos del significado; Matemáticas escolares; Álgebra; Funciones polinómicas.

\begin{abstract}
This document covers the main aspects for the design of research that evaluates the personal meanings of ninth grade students in the construction concept of linear function. Start with the structural axes of the thesis, the theoretical review describes the theory and basis of work onto-semiotic approach Cognition and Mathematics Instruction.

In the last section, there is the research proposal under Qualitative research method and outlines the steps that will be taken into account: starting from the definition of the theoretical approach, implementation of a sequence of activities, building characterization and analysis tools , triangulation of the analysis obtained, until the final report.
\end{abstract}

Keywords: ontosemiotic approach, Object mathematician institutional and personal facets; elements meaning; school Math, Algebra, Polynomial functions.

1 Artículo de Investigación.

2 Universidad Distrital Francisco José de Caldas. Bogotá, Colombia. Contacto: fntorresz@gmail.com

3 Universidad Distrital Francisco José de Caldas. Bogotá, Colombia. Contacto: jolurduy@udistrital.edu.co 


\section{Resumo}

Este documento aborda os principais aspectos para o projeto de pesquisa que avalia os significados pessoais dos alunos do nono no conceito de construção da função linear. Comece com os eixos estruturantes da tese, a revisão teórica descreve a teoria ea base de trabalho onto-semiótico abordagem Cognição e Instrução Matemática.

Na última seção, há a proposta de investigação sobre método de pesquisa qualitativa e descreve os passos que serão tomados em conta: a partir da definição da abordagem teórica, a implementação de uma seqüência de atividades, construindo caracterização e ferramentas de análise , a triangulação da análise obtida, até que o relatório final.

Palavras-chave: abordagem ontosemiótico, matemático aspectos institucionais e pessoais objetos, elementos de significado; Math escola, Álgebra, funções polinomiais.

\section{Introducción}

En la revisión bibliográfica, se encuentran experiencias de investigación en torno a la función y función lineal realizadas por Azcárate (1996), Higueras (1998) y García (1997) entre otros, donde se reporta que los estudiantes tienen la dificultad de establecer e identificar, el paso de la representación verbal a la representación escrita, no permitiendo el paso a otro tipo de representación (tabular, gráfica y simbólica) y una vaga idea de la noción de función lineal.

Se evidencia poco desarrollo de trabajos o herramientas para la realización de análisis relacionados con la noción de función lineal (uso de sus representaciones), a nivel de la básica a partir del Enfoque Ontosemiótico (EOS) propuesto por Godino (2001-2008) y desarrolladas para nuestro medio por (Lurduy, 2005-2011), que posibiliten una mejor comprensión y posibilidades de actuación didáctica de los profesores de la educación básica en Colombia.

Por lo anterior y bajo este marco, es importante, pertinente y viable llevar a cabo una caracterización y análisis de los significados personales de los estudiantes de grado $9^{\circ}$ en el trabajo con la función lineal y sus representaciones, en términos de significados personales declarados, evaluados y de referencia y sus relaciones con significados institucionales de referencia, pretendidos, implementados y evaluados.

\section{Pregunta orientadora}

¿Cómo analizar los significados personales de los estudiantes de grado $9^{\circ}$, en su construcción de conocimiento matemático, cuando abordan un proceso de estudio en el aula, sobre las representaciones de la función lineal?

\section{Enfoque de la investigación}

En trabajos anteriores, Godino y Colaboradores $(1991, \ldots .2006)$ han desarrollado un conjunto de nociones teóricas que configuran un enfoque ontológico-semiótico de la cognición e instrucción matemática, en el que se le asigna un papel central al lenguaje, a los procesos de comunicación e interpretación y a la variedad de objetos que se ponen en juego en la enseñanza y el aprendizaje de las matemáticas (Godino y Batanero, 1994; Godino 2002).

La motivación de estos autores para la elaboración de las nociones introducidas (significados institucionales y personales, entendidos, como sistemas 
de prácticas, objetos emergentes, dualidades cognitivas, función semiótica) fue progresar en la articulación de diversos modelos teóricos existentes en Didáctica de las Matemáticas, tales, como la Teoría de Situaciones Didácticas (Brousseau, 1986, 1997), Teoría de los Campos Conceptuales (Vergnaud, 1990), Teoría Antropológica (Chevallard, 1992, 1999).

Se presenta, así, a continuación el enfoque ${ }^{4}$ base para llevar a cabo la investigación, que se utiliza como referente base para realizar el análisis y caracterización la evaluación de los significados personales en una secuencia de actividades.

Figura 1: Enfoque Onto-Semiótico del

Conocimiento y la Instrucción Matemática (EOS)

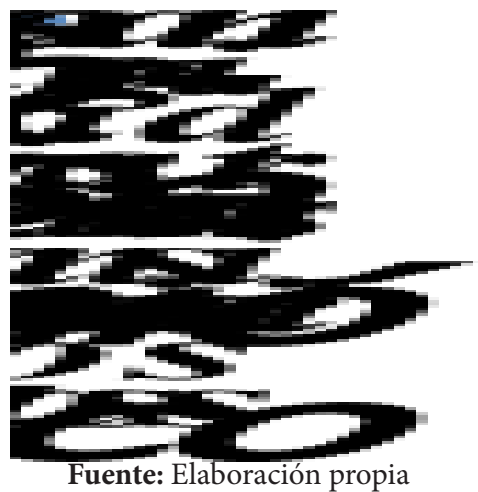

El punto de partida del EOS es la formulación de una ontología de objetos matemáticos que tiene en cuenta el triple aspecto de la matemática como actividad de resolución de problemas, socialmente compartida, como lenguaje simbólico y sistema conceptual lógicamente organizado. Tomando como noción primitiva la de situación-problemática, se definen los conceptos teóricos de práctica, objeto (personal e institucional) y significado, con el fin de hacer patente y operativo, por un lado, el triple carácter de la matemática a que hemos aludido, y por otro, la génesis personal e institucional del conocimiento matemático, así como su mutua interdependencia.

\section{Aspectos metodológicos}

Esta propuesta de investigación se enmarca dentro de la investigación cualitativa, que de acuerdo con Vera (s.f.) "estudia la calidad de las actividades, asuntos, medios, materiales o instrumentos en un determinado problema" (p. 1). Este tipo de investigación se basa en el estudio de la realidad, y se interesa más en saber cómo se da la dinámica o cómo ocurre el proceso de en que se da el asunto o problema.

Etapas de la Investigación. La propuesta de investigación se compromete en cuatro grandes etapas de desarrollo, que a continuación se describen:

Etapa 1: Revisión Bibliográfica. Durante esta etapa se implementaran tres fases: Construcción teórica y revisión bibliográfica, Apropiación teórica y Definición de anteproyecto.

Etapa 2: Apropiación Teórica. Esta etapa se implementará en cinco fases:

Etapa 3: Aplicación metodológica de la investigación. En esta etapa se examinan cuatro fases:

Etapa 4: Resultados y conclusiones. Está etapa se implementará en tres fases.

Población y Contexto de Aplicación. La investigación será dirigida a estudiantes de un curso de grado noveno $\left(9^{\circ}\right)$, del colegio distrital Fernando González Ochoa jornada Mañana, ubicado en la localidad de Usme, con un numero de 35 estudiantes, cuyas edades oscilan entre los 13 y 17 años, los cuales tienen una intensidad horaria en Matemáticas de 4 horas semanales.

Para el desarrollo de la descripción y análisis de los significados personales, se indagara inicialmente el grupo en general y en la siguiente fase se seleccionaron a tres grupos de estudiantes, de acuerdo al desempeño realizado en las actividades anteriores a la indagada, en términos de sus declaraciones y argumentos utilizados (alto, medio y bajo).

\section{Recursos}

Secuencia de Actividades. Es la herramienta que configura la propuesta para la construcción de la noción de función lineal haciendo uso de las

4 Se entiende por enfoque el desarrollo teórico con el cual se va a llevar a cabo la investigación. 
representaciones: verbal, escrita, tabular, gráfica y simbólica.

Estudiantes. Son los protagonistas de la puesta en escena de la secuencia de actividades, y a partir de las devoluciones de estos es donde se hace el análisis en torno a los significados personales de los estudiantes.

Portafolios. En estos, los estudiantes consignaran cada una de las representaciones funcionales realizadas a lo largo de la construcción de Mi Zoom, además de ser una manera llevar un seguimiento de lo que se va logrando con cada una de las actividades planteadas en la secuencia propuesta.

Grabación de la Actividad de Validación. Punto de vista neutral de la realización y de los temas vistos en la actividad "Mi Zoom", con el fin de tener una más fina mirada de lo realizado por el profesor y los estudiantes.

Estructura Secuencias de Actividades. Después del pilotaje, restructuración y validación de la secuencia, se presenta la propuesta desarrollada por Torres y Nieto (2008), en la fase de aplicación y valoración de su investigación.

Grafica 2

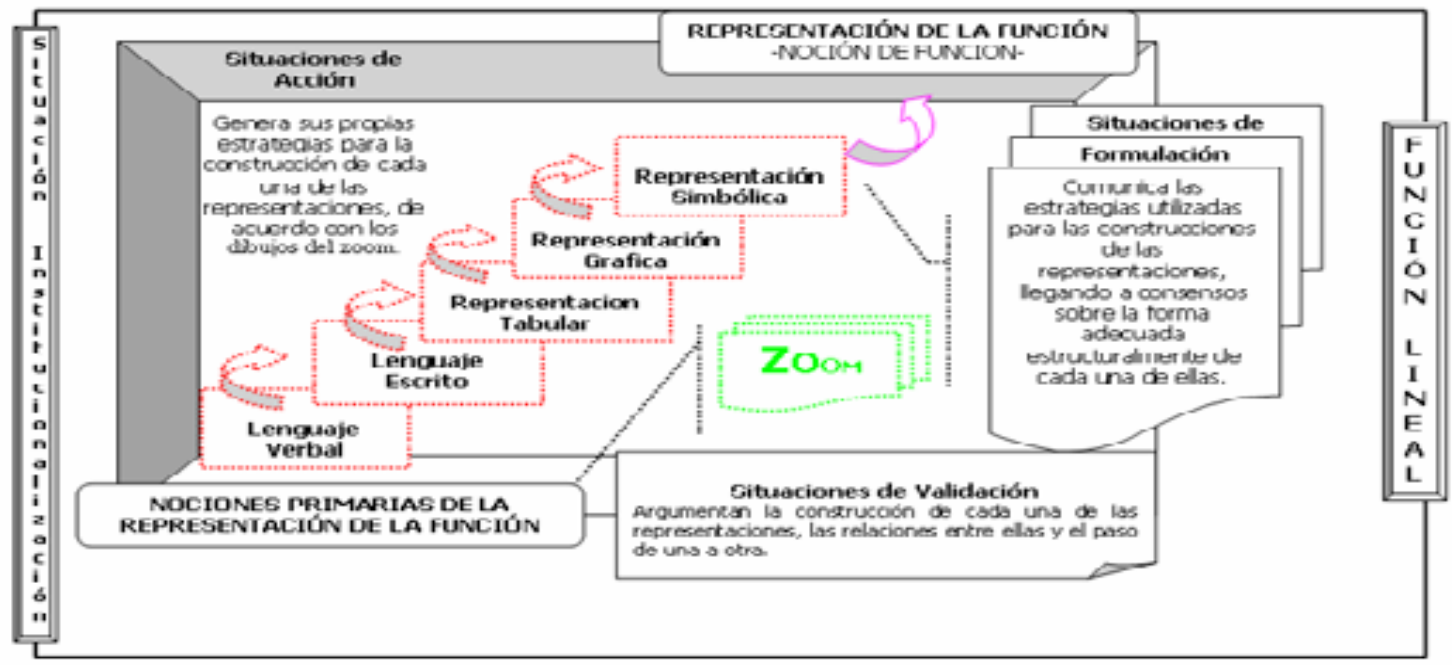

Fuente: Elaboración propia

\section{Referencias}

Azcárate, C Y otros. (1996). Funciones y Gráficas. Editorial Síntesis. Octubre.

García, G Y otros. (1997). Cuadernillo didáctico. Hacia la noción de función como dependencia. Universidad Pedagógica Nacional. Bogotá.

Godino, J. D. (1999). Implicaciones metodológicas de un enfoque semiótico-antropológico para la investigación en didáctica de las matemáticas. En T. Ortega (Ed.). Actas del III Simposio de la SEIEM (pp. 196-212). [Recuperable en, http://www.ugr.es/local/jgodino/semiotica.htm].
Godino J. D. (2003). Teoría de las funciones semióticas. Un enfoque ontológico-semiótico de la cognición e instrucción matemática. Departamento de Didáctica de la matemática. Universidad de Granada. Disponible en Internet: URL: http://www.urg.es/local7jgodino7indice_tfs.htm.

Lurduy, O. (2012) El tetraedro didáctico como modelación de los procesos de estudio de las matemáticas en el aula. DIE-UD. Bogotá, Colombia

Ruiz Higueras, L. (1998). La noción de función: análisis epistemológico y didáctico. Universidad de Jaén. Jaén. 